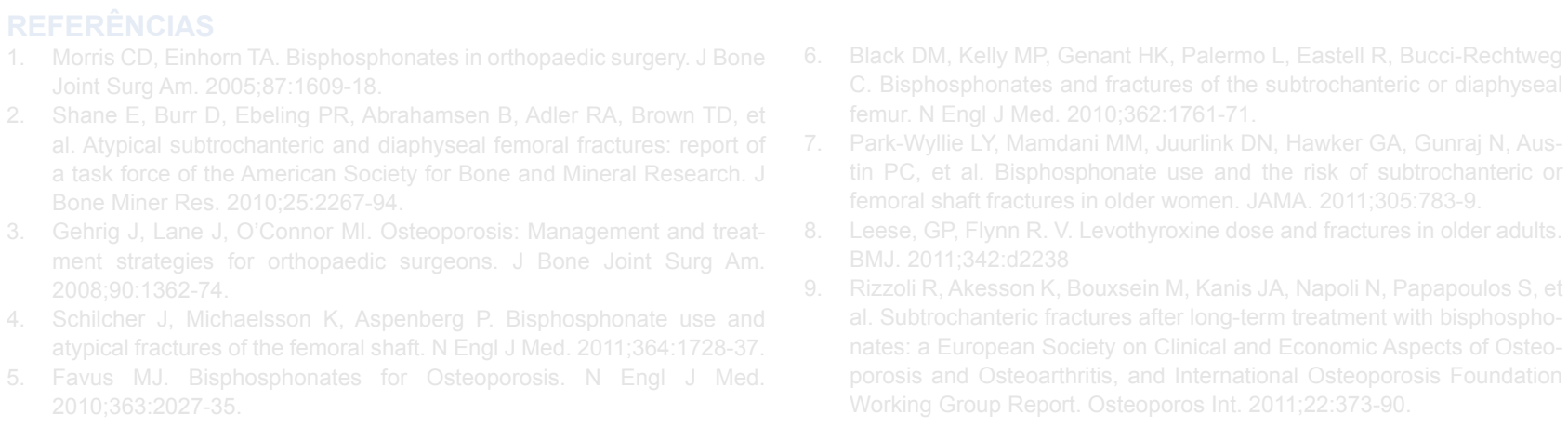

\title{
Hemangioma Hepático Infantil Gigante: Que Opções Terapêuticas?
}

\author{
Giant Infantile Hepatic Hemagioma: Which Terapeutic Options?
}

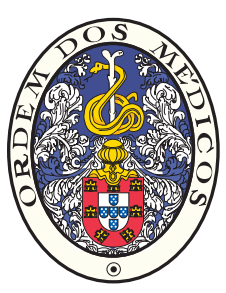

\author{
Cristina GONÇALVES ${ }^{1}$, Luísa LOBO², Rui ANJOS ${ }^{3}$, Carlos SALGUEIRO4, Ana Isabel LOPES ${ }^{1}$ \\ Acta Med Port 2013 Nov-Dec;26(6):750-754
}

\section{RESUMO}

Os hemangiomas hepáticos infantis constituem o terceiro tumor hepático mais frequente na criança e o mais frequente antes dos seis meses. As opções terapêuticas são determinadas pela apresentação clínica, devendo ser individualizadas. Apresenta-se o caso de uma criança actualmente com quatro anos de idade, com diagnóstico neonatal de volumosa malformação hepática vascularizada, com critérios imagiológicos compatíveis com hemangioma hepático infantil. Destaca-se a ocorrência inicial de Síndrome de KasabachMerrit (trombocitopenia, anemia) e insuficiência cardíaca que resolveram espontaneamente. Ao longo do período de seguimento, o estudo imagiológico evolutivo (ecografia, doppler ressonância magnética e tomografia computorizada com administração de contraste endovenoso) confirmou a hipótese de $\mathrm{HHI}$ ao permitir o mapeamento vascular detalhado. A partir do primeiro ano de vida, constatou-se evolução favorável com redução progressiva da massa. Embora se tenha mantido atitude conservadora, a melhor abordagem e intervenção nesta entidade, permanece controversa. Salientam-se as particularidades deste caso, discutindo a abordagem com melhor relação custo-benefício.

Palavras-chave: Criança; Hemangioendotelioma; Neoplasias do Fígado; Portugal.

\section{ABSTRACT}

Infantile Hepatic Hemagioma is the third most frequent liver tumor in children and the most common below 6 months of age. Therapeutic options depend on clinical manifestations and should be tailored on an individual patient basis. We present the case of a 4 year old boy with neonatal diagnosis of large vascularized liver tumor with imagiological criteria of Infantile Hepatic Hemagioma. We highlight the occurrence of heart failure and Kasabach-Merrit syndrome (thrombocytopenia, anemia) that have spontaneously regressed. During follow up, sequential imaging (ultrasound with Doppler, magnetic resonance imaging, dynamic contrast enhancement computed tomography) confirmed the hypothesis of $\mathrm{IHH}$, allowing vascular mapping of the lesion. From the first year on, we observed a favorable course with progressive tumor regression. In the present case, a conservative approach has been maintained, but the best therapeutic option remains unclear. We highlight the specific features of this case, discussing the most cost - effective approach.

Keywords: Child; Hemangioendothelioma; Liver Neoplasms; Portugal.

\section{INTRODUÇÃO}

O HemangiomaHepático Infantil $(\mathrm{HHI})$ é uma entidade rara, ${ }^{1,2}$ que se apresenta com maior frequência nos primeiros meses de vida ${ }^{3}$ e cujas manifestações clínicas, o prognóstico e a terapêuticas dependem da localização e do tamanho do tumor.

Os autores relatam o caso de uma criança com diagnóstico de $\mathrm{HHI}$ no período neonatal, que pelas suas dimensões suscitou discussão das opções terapêuticas actual-

mente disponíveis, numa perspectiva de risco-benefício.

\section{CASO CLÍNICO}

Apresenta-se o caso de um rapaz, actualmente com quatro anos, fruto de gestação vigiada, com serologias negativas, ecografias pré-natais sem alterações; cesariana às 36 semanas por colestase gravídica. Ao segundo dia de vida, por Síndrome de Difiuldade Respiratória (SDR),

1. Unidade de Gastrenterologia Pediátrica. Departamento de Pediatria. Hospital de Santa Maria. Centro Académico de Medicina de Lisboa. Lisboa. Portugal.

2. Serviço de Imagiologia Geral. Hospital Santa Maria (Centro Hospitalar Lisboa Norte). Lisboa. Portugal.

3. Serviço de Cardiologia Pediátrica. Hospital Santa Cruz (Centro Hospitalar Lisboa Oeste). Carnaxide. Portugal.

4. Clínica Nascer e Crescer. Barreiro. Portugal.

Recebido: 01 de Janeiro de 2013 - Aceite: 21 de Abril de 2013 | Copyright @ Ordem dos Médicos 2013 


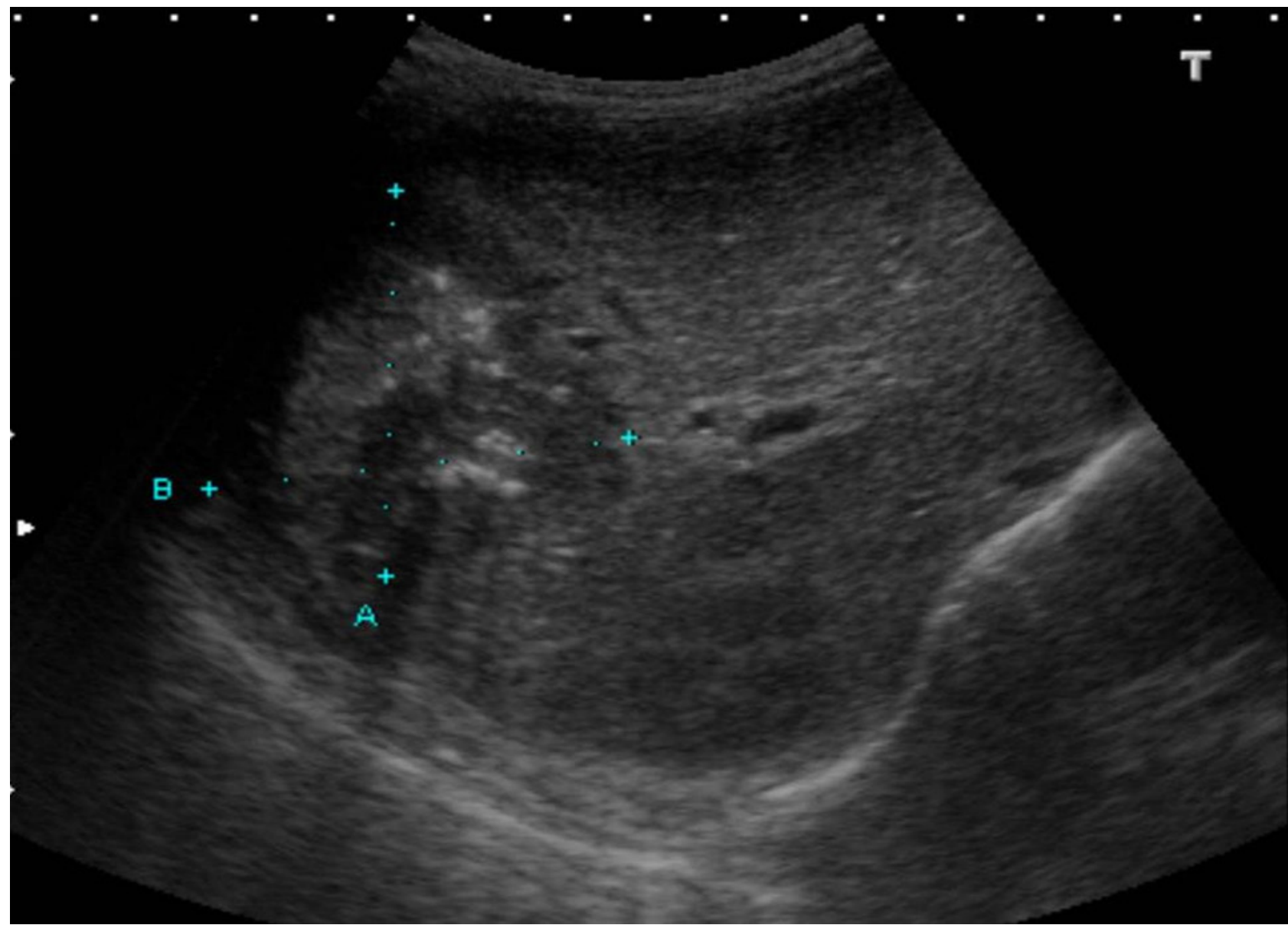

Figura 1 - Ecografia hepática, plano transversal do lobo direito do fígado, que mostra massa sólida heterogénea com área central hiperecogénea e focos calcificados no seu interior.

hepatomegália, icterícia e sopro cardíaco foi internada na Unidade de Cuidados Intensivos Neonatais. Analiticamente apresentava anemia $(\mathrm{Hb}: 11,5 \mathrm{~g} / \mathrm{dL})$, trombocitopenia $\left(60000 / \mathrm{mm}^{3}\right)$, hiperbilirrubinémia não conjugada (bilirrubina total/ directa: 9,2/2,2 $\mathrm{mg} / \mathrm{dL}$ ), sem alterações das enzimas hepáticas e da coagulação - dados considerados compatíveis com S. Kasabach-Merritt; na observação cardiológica: insuficiência cardíaca ligeira, sem cardiopatia estrutural. Efectuou ecografia abdominal evidenciando hepatomegália condicionada por massa no lobo direito $(8 \times 6 \times 4 \mathrm{~cm})$, com ecostrutura heterogénea, focos hiperecogéneos (calcificações) e exuberante vascularização periférica no Doppler; aumento do calibre do tronco celíaco e da artéria hepática, com redução do calibre da aorta abdominal após a emergência do tronco celíaco. (Fig.s 1 e 2). Foram colocadas as hipóteses de diagnóstico de $\mathrm{HHI}$ versus malformação arterio-venosa. Salienta-se a-fetoproteína: $69459 \mathrm{ng} / \mathrm{mL}$ (valor da primeira semana de vida) e antigénio carcino-embrionário: $4,7 \mathrm{ng} / \mathrm{mL}$ (normal $<5,2 \mathrm{ng} / \mathrm{mL}$ ).

Ao $10^{\circ}$ dia de vida efectuou ressonância magnética nuclear (RMN) abdominal que favoreceu a hipótese de $\mathrm{HHI}$ : volumosa massa hepática heterogénea $(7 \times 6 \times 5 \mathrm{~cm})$, de contornos bem definidos, irregulares e lobulados, com sinal hipointenso em T1 e hiperintenso em T2; aumentado cali- bre do tronco celíaco, da artéria hepática, das veias supra-hepáticas média e direita e da veia cava inferior.

Decidida atitude expectante com melhoria gradual do SDR, da icterícia, da anemia e trombocitopénia que se voltaram a manifestar após a alta, com um mês de vida $(\mathrm{Hb}$ : $7,2 \mathrm{~g} / \mathrm{dL}$, plaquetas: $\left.172000 / \mathrm{mm}^{3}\right)$, assistindo-se a nova regressão espontânea. Aos quatro meses mantinha massa palpável no hipocôndrio e flanco direitos, limites bem definidos, consistência aumentada e superfície lisa com sopro vascular audível. Nesta fase, a avaliação cardíaca foi normal.

Manteve-se seguimento clínico e ecográfico, e aos dois anos efectuou tomografia computorizada (TC) com contraste, mostrando lesão sólida hipervascularizada com calcificações e padrão de captação periférico e centrípeto, tornando-se homogénea nas fases tardias, compatível com HHI. (Fig.s 3 e 4).

Ao longo do seguimento, permaneceu assintomático, com boa progressão estaturo-ponderal e adequado desenvolvimento psico-motor, mantendo massa hepática palpável, embora de dimensões progressivamente.

A última ecografia (aos anos anos e seis meses) evidenciou ligeira redução da massa $(6,5 \times 5,5 \mathrm{~cm})$, com menor exuberância do fluxo vascular. 


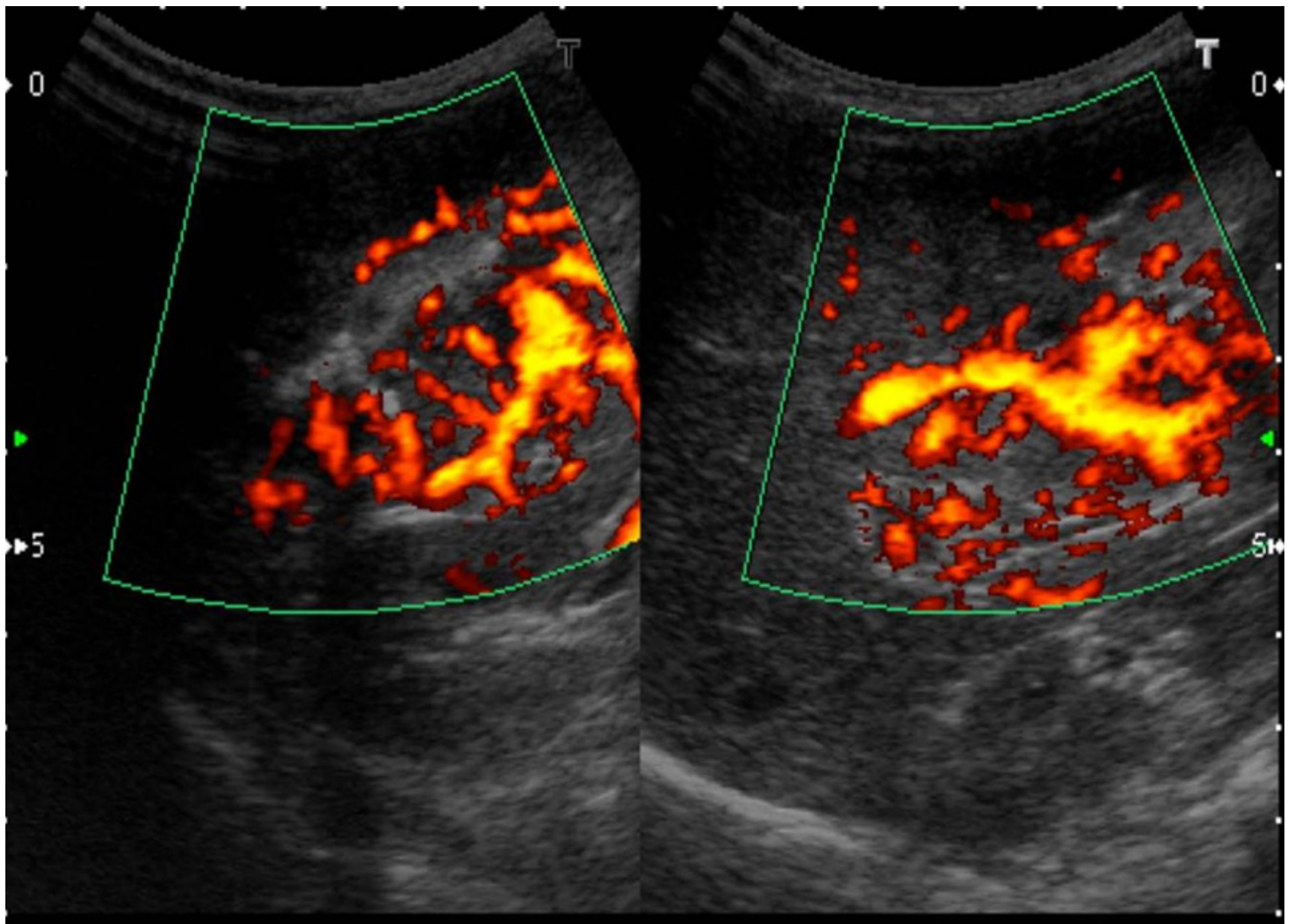

Figura 2 - Ecografia com Doppler a cor mostrando que a lesão é hipervascularizada, com vasos de maior calibre à sua periferia.

\section{DISCUSSÃo}

O HHI é um tumor vascular de características histológicas semelhantes aos hemagiomas cutâneos. ${ }^{4}$ É o segundo tumor hepático mais comum abaixo dos dois anos de vida e o mais comum abaixo dos seis meses. ${ }^{5}$ Samuel e Spitz descreveram uma série de lactentes com $\mathrm{HHI}$, em que a maioria se manifestou abaixo dos três meses de vida, ${ }^{3}$ tal como no caso reportado. A apresentação clínica depende das dimensões e da localização do tumor, permanecendo assintomático no caso de tumores de pequenas dimensões. A sintomatologia mais frequentemente referida na literatura ${ }^{3,6}$ foi a hepatomegália seguida da massa/distensão abdominal, a associação com hemangiomas cutâneos, a coagulopatia de consumo e a insuficiência cardíaca, esta última com elevada incidência e significativa morbi-mortalidade associada. ${ }^{7}$ A S. Kasabach-Merritt, caracterizada pela associação de anemia, trombocitopénica e/ou coagulopatia de consumo, tem uma incidência de 20 a $75 \%$ nos $\mathrm{HHI}$ no período neonatal ${ }^{3,8}$ ascendendo as taxas de mortalidade aos $40 \%{ }^{9}$ No presente caso, apesar da exuberância da apresentação clínica no período neonatal, verificou-se uma evolução favorável sob atitude conservadora.

O diagnóstico histológico não é geralmente necessário. A biopsia, cirúrgica ou mesmo guiada por ecografia, é um procedimento com risco hemorrágico significativo em tumores vasculares, devendo a sua indicação ser muito ponderada.

Neste caso, os aspectos imagiológicos foram cruciais

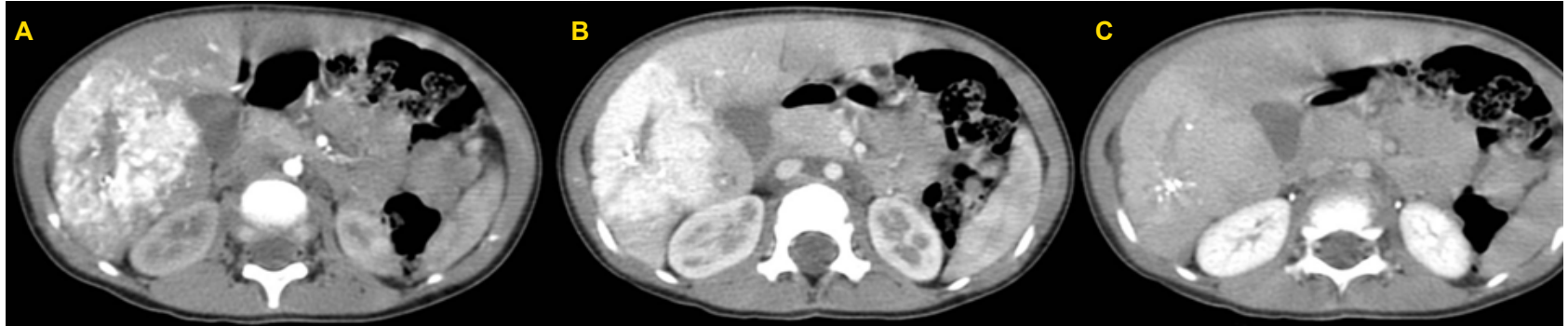

Figura 3 - TC com contraste endovenoso na fase arterial (A), portal (B) e tardia (C) mostrando massa sólida hipervascularizada no lobo hepático direito, com padrão de captação de contraste centrípeto característico de hemangioma: ganho periférico intenso inicial, do tipo algodonoso, com progressão para o centro tornando-se homogénea e quase isodensa com o parênquima. 


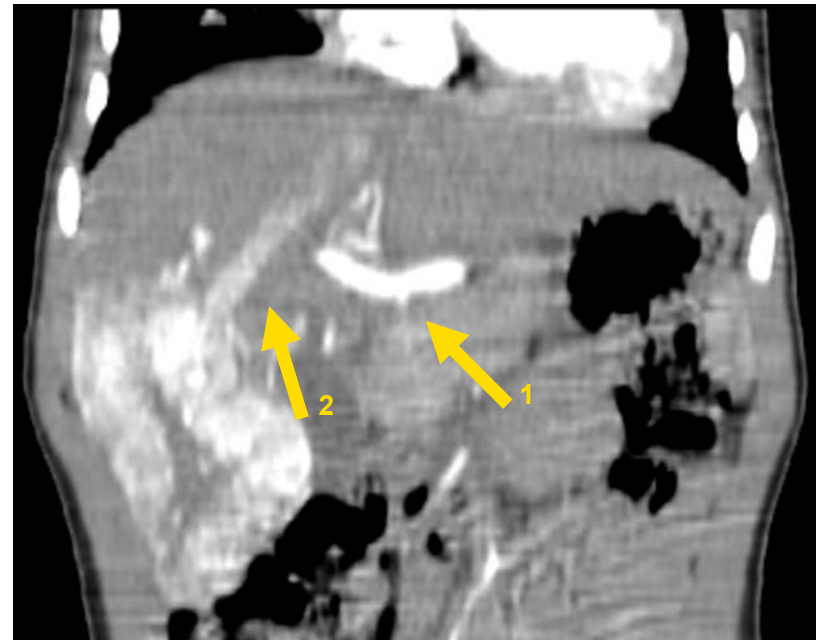

Figura 4 - Reconstrução coronal da fase arterial mostrando aspecto da lesão no lobo direito irrigada por vários pedículos da artéria hepática, com elevado calibre da artéria hepática (seta 1) e de drenagem para as veias supra-hepáticas média e direita com aumento do seu calibre (seta 2).

para o diagnóstico. Ecograficamente evidenciou-se massa hepática de grandes dimensões, heterogénea incluindo focos hiper, iso e hipoecogénicos ${ }^{10}$ com intensa vascularização no estudo Doppler, embora menos pronunciada na região central. No estudo por RMN, salienta-se boa delimitação e hipointensidade da massa em relação ao restante fígado em T1 com sinal hiperintenso em T2. ${ }^{11}$ No caso da TC, a injecção de contraste é determinante para a caracterização deste tipo de massas, em virtude de apresentarem um padrão de captação de contraste centrípeto característico, com captação periférica algodonosa na fase arterial e progressão central numa fase mais tardia venosa. ${ }^{12}$

A terapêutica deverá ser individualizada de acordo com as dimensões, a localização do tumor e a condição clínica do doente, não existindo consenso sobre a melhor abordagem. ${ }^{6,13}$ Dadas as dimensões da massa e não ter ocorrido a involução esperada após o final do primeiro ano de vida e associadamente ao início da socialização com potencial risco de traumatismo abdominal e rotura, este caso suscitou discussão alargada das opções terapêuticas. Na criança assintomática com lesão única será legítimo não instituir qualquer terapêutica, mantendo monitorização clínica ${ }^{13} \mathrm{em}$ consonância com a atitude adoptada neste caso. No caso de existirem sintomas e/ou complicações não controláveis, existem várias opções disponíveis. A terapêutica farmacológica poderá incluir corticosteroides, ${ }^{14}$ interferão-alfa2a2, ${ }^{15,16}$ ou propranolol, ${ }^{17}$ com o objectivo de reduzir o leito vascular da lesão. No entanto, a relação risco-benefício destes fármacos não está bem estabelecida existindo complicações reportadas, ${ }^{16,18}$ e taxas de sucesso variáveis. ${ }^{2,15}$ A terapêutica cirúrgica poderá passar pela ressecção da massa (tumores pequenos e confinados a um lobo hepático) ou em último recurso pelo transplante hepático (massas irressecáveis). ${ }^{19}$ A embolização da artéria hepática é outra opção a ser considerada em casos de insuficiência cardíaca grave e descompensada, embora com resultados contraditórios. ${ }^{20}$

Após os primeiros meses de vida em que o impacto na função cardíaca e pulmonar são determinantes, na maioria dos casos os HHI têm um bom prognóstico, assistindo-se a um regressão a partir do final do primeiro ano de vida, ${ }^{2}$ provavelmente por fenómenos de trombose e cicatrização. ${ }^{20}$ No nosso doente, após uma fase em que o tamanho da massa permaneceu estável, esta acabou por revelar o perfil evolutivo característico.

De realçar a necessidade de manter o seguimento clínica e imagiológico a longo prazo, dada a incerteza do potencial de malignização, existindo relatos de $\mathrm{HHI}$ que posteriormente se apresentaram como angiossarcomas. ${ }^{3}$

\section{CONCLUSÃO}

Em resumo, apresenta-se um caso particular de $\mathrm{HHI}$, pelas suas dimensões e comportamento, que condicionaram a discussão das diferentes opções terapêuticas, enfatizando-se a importância da abordagem multidisciplinar e de consenso sobre a melhor estratégia.

\section{CONFLITOS DE INTERESSE}

Os autores declaram não existir conflitos de interesse.

\section{FONTES DE FINANCIAMENTO}

O presente trabalho não foi objecto de qualquer bolsa/ financiamento para a sua realização.

\section{REFERÊNCIAS}

1. Burtelow M, Garcia M, Lucile S, Cox K, Berquist W, Kerner J. Hepatic Infantile Hemagioendothelioma with unusual manifestations. J Pediatr Gastroenterol Nutr. 2006;42:109-13.

2. Zenge JP, Fentol L, Lovell MA, Grover TR. Case report: Infantile Hemagioendothelioma. Curr Opin Pediatr. 2002;14:99-102.

3. Samuel M, Spitz L. Infantile hepatic hemagioendothelioma: the role of surgery. J Pediatr Surg. 1995;30:1425-9.

4. Mo JQ, Dimashkieh HH, Bove KE. Glut1 endothelial reactivity distinguishes hepatic infantile hemangioma from congenital hepatic vascular malformation with associated capillary proliferation. Hum Pathol. 2004;35:200-9.

5. Ishak KG, Goodman ZD, Stocker JT. Tumors of the liver and intrahepatic bile ducts. Atlas of Tumor Pathology. Washington: Armed Forces Institute of Pathology; 2001.

6. Daller JA, Bueno J, Gutierrez J, Dvorchik I, Towbin RB, Dickman PS, et al. Hepatic hemagioendothelioma: clinical experience and management strategy. J Pediatr Surg. 1999; 34:98-105.

7. Rochini AP, Rosenthal A, Issenberg HJ, Nadas AS. Hepatic Hemagioendothelioma: hemodynamic observations and treatment. Pediatrics. 1976;57:131-5.

8. Shim WKT. Hemangiomas of infancy complicated by thrombocytopenia. Am J Surg. 1968;116:896-906.

9. Fishman SJ, Mulliken JB. Hemangiomas and vascular malformations of infancy and childhood. Pediatr Clin N Am. 1993;40:1177-200.

10. Ehren $\mathrm{H}$, Mahour $\mathrm{GH}$, Isaacs $\mathrm{H}$ Jr. Benign liver tumors in infancy and childhood. Report of 48 cases. Am J Surg. 1983;145:325-9.

11. Kassarjian A, Zurakowski D, Dubois J, Paltiel HJ, Fishmnan SJ, Burrows PE. Infantile hepatic hemangiomas: clinical and imaging findings and their correlation with therapy. AJR Am J Roentgenol. 2004;182:785-95.

12. Lucaya J, Enriquez G, Amat L, Gonzalez-Rivero MA. Computed tomography of infantile hepatic hemangioendothelioma. AJR Am J Roentgenol. 1985;144:821-6

13. Christison-Legay ER, Burrows PE, Alomari A, Dubois J, Kozakewich HP, Lane TS, et al. Hepatic hemangiomas: subtype classification and 
development of a clinical practice algorithm and registry. J Pediatr Surg. 2007;42:62-7.

14. Park EA, Seo JW, Lee SW, Choi HY, Lee SJ. Infantile hemangioendothelioma treated with high dose methylprednisolone pulse therapy. J Korean Med Sci. 2001;16:127-9.

15. Boon LM, Burrows PE, Paltiel HJ, Lund DP, Ezekowitz AB, Folkman J, et al. Hepatic vascular anomalies in infancy: a twenty seven year experience. J Pediatr. 1996;129:346-54.

16. Woltering MC, Robben S, Egeler RM. Hepatic hemagioendothelioma of infancy; Treatment with interferon alpha. J Pediatr Gastroenterol Nutr. 1997;24:348-51.

17. Sans V, de la Roque ED, Berge J, Grenier N, Boralevi F, Mazereeuw-
Hautier J, et al. Propranolol for severe infantile hemangiomas: follow-up report. Pediatrics. 2009;124:e423-31.

18. Weber TR, Connors RH, Tracy TF Jr, Bailey PV. Complex hemangiomas of infancy and childhood. Arch Surg. 1990;125:1017-21.

19. Achilleos OA, Buist LJ, Kelly DA, Raafat F, McMaster P, Mayer AD, et al Unresectable hepatic tumors in childhood and the role of liver transplantation. J Pediatr Surg. 1996;31:1563-7.

20. Nguyen L, Shandling B, Stephens C. Hepatic Hemangioma in Childhood: medical management or surgical management? J Pediatr Surg. 1982;17:576-9.

21. Davenport M, Hansen L, Heaton ND, Howard ER. Hemangioendothelioma of the liver in infants. J Pediatr Surg. 1995;30:44-8. 


\section{Hemangioma Hepático Infantil Gigante: Que Opções Terapêuticas? \\ Acta Med Port 2013:26:750-754}

Publicado pela Acta Médica Portuguesa, a Revista Científica da Ordem dos Médicos

Av. Almirante Gago Coutinho, 151

1749-084 Lisboa, Portugal.

Tel: +351218428 215

E-mail: submissao@actamedicaportuguesa.com

www.actamedicaportuguesa.com

ISSN:0870-399X | e-ISSN: 1646-0758

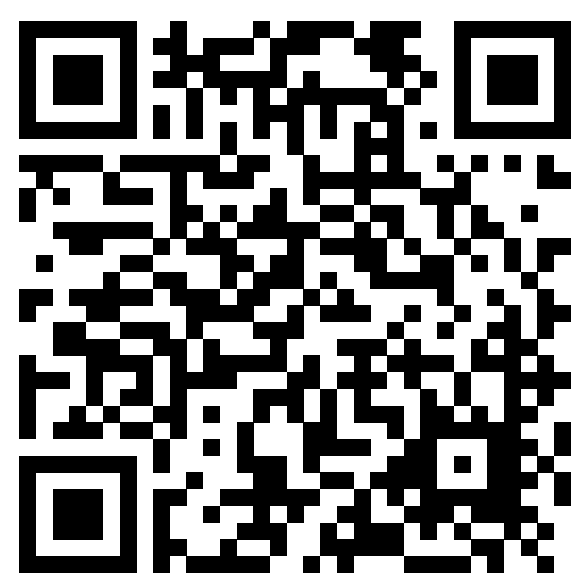

